\title{
Green and efficient aza-Michael additions of aromatic amines to $\alpha, \beta$-unsaturated ketones catalyzed by DBU based task-specific ionic liquids without solvent
}

\author{
An-Guo Ying, ${ }^{\text {a,b }}$ Li-Min Wang, ${ }^{\mathrm{b}}$ Hong-Xia Deng, ${ }^{\mathrm{c}}$ Jian-Hui Chen, ${ }^{\mathrm{b}}$ Xin-Zhi Chen, ${ }^{\text {** }}$ and \\ Wei-Dong $\mathrm{Ye}^{\mathrm{b}} *$ \\ ${ }^{a}$ Department of Chemical Engineering Zhejiang University, Hangzhou 310027, P R China \\ ${ }^{b}$ Zhejiang Medicine Co., LTD. Xinchang Pharmaceuticals Factory, Xinchang 312500, P R China \\ ${ }^{c}$ Zhejiang Chemical Industry Research Institute, Hangzhou 310023, P R China \\ E-mail:xzchen@,zju.edu.cn,yewd@xcpharma.com
}

\begin{abstract}
The DBU (1,8-Diazabicyclo[5.4.0]undec-7-ene) derived task-specific ionic liquids [DBU][Lac] (1,8-diazabicyclo[5.4.0]-undec-7-en-8-ium lactate), [DBU][Ac] (1,8-diazabicyclo[5.4.0]-undec7-en-8-ium acetate) and [DBU][Tfa] (1,8-diazabicyclo[5.4.0]-undec-7-en-8-ium trifluoroacetate) were synthesized. Among the three novel ionic liquids, [DBU][Lac] was found to have the highest catalytic activity for aza-Michael addition of aromatic amines to $\alpha, \beta$-unsaturated ketones. The protocol can give good to excellent products yields and has advantages such as readily work-up and good reusability, which makes this method quite attractive.
\end{abstract}

Keywords: DBU, ionic liquid, aza-Michael addition, aromatic amines, $\alpha, \beta$-unsaturated ketones

\section{Introduction}

The aza-Michael addition is one of the widely used reactions for carbon-nitrogen bond formation in modern organic synthetic chemistry. Conjugate reaction of various amines with $\alpha, \beta$ unsaturated carbonyl compounds provides $\beta$-amino carbonyl ingredients, which have attracted great attention for their use as key intermediates of anticancer agents, antibiotics and other drugs. ${ }^{1}$ Generally, aza-Michael additions have been catalyzed by strong bases and acids, and some side reactions occurred. Therefore, chemical researchers have paid more attentions to the development of more mild catalytic systems for the aza-conjugate reaction. A number of alternative methodologies using Lewis acidic catalysts such as $\mathrm{PtCl}_{4} \cdot 5 \mathrm{H}_{2} \mathrm{O},{ }^{2 \mathrm{a}} \mathrm{Cu}(\mathrm{OTf})_{2},{ }^{2 \mathrm{~b}} \mathrm{InCl}_{3},{ }^{2 \mathrm{c}}$ $\mathrm{Yb}\left(\mathrm{OTf}_{3}\right),{ }^{2 \mathrm{~d},}{ }^{2 \mathrm{e}} \mathrm{LiClO}_{4}{ }^{2 \mathrm{f}} \mathrm{Bi}\left(\mathrm{NO}_{3}\right),{ }^{2 \mathrm{~g}} \mathrm{FeCl}_{3} \cdot 6 \mathrm{H}_{2} \mathrm{O},{ }^{2 \mathrm{~h}} \mathrm{CeCl}_{3} \cdot 7 \mathrm{H}_{2} \mathrm{O},{ }^{2 \mathrm{i}} \mathrm{ZnO}^{2 \mathrm{j}} \mathrm{MgO}$ assisted by microwave irradiation, ${ }^{2 \mathrm{k}}$ silica supported perchloric acid $^{21}$ and sulfated zirconia ${ }^{2 \mathrm{~m}}$ have been reported. Recently, silica gel in acetonitrile, ${ }^{3 a} \beta$ - cyclodextrin in water, ${ }^{3 b}$ PEG (MW 400) and 2- 
hydroxyethylalmmonium formate using as reaction medium as well as reaction catalyst, ${ }^{3 \mathrm{c}, 3 \mathrm{~d}}$ boric acid in water, ${ }^{3 \mathrm{e}}$ and even using water as solvent without any catalyst ${ }^{3 \mathrm{f}}$ had been employed as efficient reaction systems for the aza-Michael addition of amines to electron-deficient olefins. However, many of the above methods suffered from some drawbacks, for example, the requirement for a large excess of reagents, substrate-selective for some catalysts, and often involvement of some toxic solvents such as 1,2-dichloroethane or acetonitrile. Moreover, because of the inertness of arylamines compared with aliphatic amines, most of the above methods are not successful with aromatic amines. Duan and co-workers used CAN (ceric ammonium nitrate) as effective promoter for aza-Michael addition of aromatic and aliphatic amines to $\alpha, \beta$-unsaturated electrophiles in the absence of solvent under ultrasound irradiation. ${ }^{4}$ Very recently, Bhanage et al. reported $\mathrm{Y}\left(\mathrm{NO}_{3}\right)_{3} \cdot 6 \mathrm{H}_{2} \mathrm{O}$ catalyzed aza-conjugate reaction of weakly nucleophilic aromatic amines with various Michael acceptors like esters, nitriles and amides under solvent-free conditions. ${ }^{5}$ However, both of the two procedures utilized transition metal catalysts, which limited their applications from the economic and environmental viewpoints, and more importantly, they have not involved in the aza-Michael reaction with $\alpha, \beta-$ unsaturated ketones as Michael acceptors. Basic ionic liquid [bmim]OH (1-butyl-3methylimidazolium hydroxide) was first introduced as catalyst for the conjugate reaction of aromatic amines and to our disappointment, relatively long reaction times were required. ${ }^{6}$ Thus, the development of an efficient and green protocol for aza-Michael addition of aromatic amines to electron-deficient ketones still remains a challenging task and is highly desirable.

1,8-Diazabicyclo[5.4.0] undec-7-ene (DBU) was found to be far superior to other tertiary amines and its applications in organic synthesis have been deeply investigated over the past decades. ${ }^{7}$ Furthermore, DBU using as catalyst/promoter has also been used in aza-Michael reaction of various aliphatic and $N$-heterocycles amines. ${ }^{8}$ As we all know, the typical disadvantages of organic bases, including unpleasant flavor and hardly recyclability generally exist in industrial process. Ionic liquids, due to their unique properties like good solvating ability, negligible vapor pressure, variable polarity, nonflammability and recyclability have been widely used as catalysts as well as reaction mediums in organic synthesis with more or less success. ${ }^{9}$ Herein we wish to develop some task-specific ionic liquids other than reported inert ionic liquids $^{10}$ derived from DBU for aza-Michael addition of aromatic amines to $\alpha, \beta$-unsaturated ketones at room temperature under solvent-free conditions.

\section{Results and Discussion}

We synthesized three DBU-based task-specified ionic liquids by simply neutralization reaction of DBU and three carboxyl acids, acetic acid, lactic acid, and trifluoroacetic acid (Scheme1). The reaction of aniline with ethyl vinyl ketone $(\mathrm{EVK})$ was selected as model to optimize the reaction conditions. First, using [bmim] OH which is highly efficient in Michael reaction of carbon nucleophiles and aliphatic amines as catalyst, ${ }^{11} 8 \mathrm{~h}$ was required for disappearance of the starting 
material (Table 1, entry 4). ${ }^{6}$ Inert ionic liquid [bmim]BF 4 (1-butyl-3-methylimidazolium tetrafluoroborate) was also tested as promoter for the reaction and only $55 \%$ yield of the desired product was obtained in $40 \mathrm{~min}$ (Table 1, entry 5). For comparison, some Lewis bases such as DMAP, $\mathrm{PPh}_{3}$, and DBU were subjected to the model reaction and bis-adduct was formed thereby decreasing the product yield (Table 1, entries 6-8). Especially, with DBU, the bis-addition product was produced as much as $32 \%$ yield while using its corresponding ionic liquids $[\mathrm{DBU}][\mathrm{Ac}],[\mathrm{DBU}][\mathrm{Lac}]$, and [DBU][Tfa] no bis-addition reaction was observed and 96-99\% yields were afforded within the same period, demonstrating the good chemo- and regioselectivity of these ionic liquids in the reaction of aniline with ethyl vinyl ketone (Table 1, entries 1-3). Among the three ionic liquids, [DBU][Lac] performed better than the other ionic liquids, [DBU][Ac] and [DBU][Tfa] (entries 1-3). So we chose [DBU][Lac] as catalyst for further investigations. A blank experiment was also carried out to demonstrate the catalytic activity of the DBU-based task-specific ionic liquids (Table 1, entry 9). To optimize the details of the reaction conditions, solvent effect was studied in the same model reaction between aniline and EVK (Table 1, entry 1). Toluene, $\mathrm{CH}_{2} \mathrm{Cl}_{2}, \mathrm{CH}_{3} \mathrm{OH}$, and $\mathrm{CH}_{3} \mathrm{CN}$ were all found to be effective media for the reaction and relatively lower reactant concentration in organic solvent than that under solvent-free conditions decreased the reaction rate. We then selected the solventfree conditions rather than using organic solvents from the environmental and reaction rate considerations.

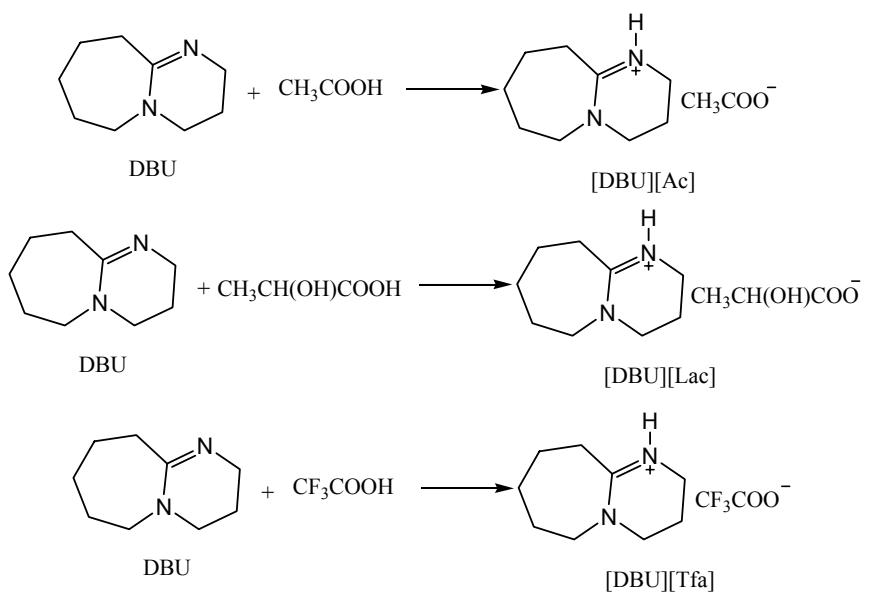

Scheme 1. Synthesis of Ionic Liquid [DBU][Ac], [DBU][Lac], and [DBU][Tfa].

With the efficient catalytic system in hand, EVK was treated with other aromatic amines. The results are summarized in Table 2 (entries 1-5). Aromatic amines with electron-donating group at bezene ring were effective nucleophiles to react smoothly with EVK and excellent yields were afforded in very short reaction time (Table 2, entries 1-2), while 2-Methyl substituted amine, due to its steric hindrance, reacted relatively slowly (entry 3). $p-\mathrm{NO}_{2}$ substituted amine could not undergo the reaction at all (entry 5). Arylamine with weakly electron-drawing substitution $\mathrm{Cl}$ 
was also tested with EVK under the above reaction conditions and $95 \%$ GC yield was obtained in $1 \mathrm{~h}$ (entry 4).

Table 1. Aza-Michael reaction of aniline with ethyl vinyl ketone under various reaction conditions $^{\mathrm{a}}$

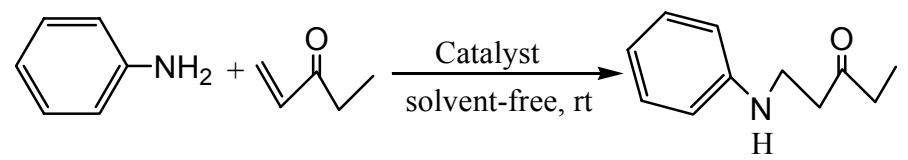

\begin{tabular}{llll}
\hline Entry & Catalyst & Time $(\min )$ & Yield $^{\mathrm{b}}(\%)$ \\
\hline 1 & {$[\mathrm{DBU}][\mathrm{Lac}]$} & 30 & $99\left(82^{\mathrm{c}}, 75^{\mathrm{d}}, 76^{\mathrm{e}}, 70^{\mathrm{f}}\right)$ \\
2 & {$[\mathrm{DBU}][\mathrm{Ac}]$} & 40 & 96 \\
3 & {$[\mathrm{DBU}][\mathrm{Tfa}]$} & 40 & 96 \\
$4^{\mathrm{g}}$ & {$[$ bmim]OH } & 480 & $98^{\mathrm{g}}$ \\
5 & {$\left[\right.$ bmim]BF $_{4}$} & 40 & 55 \\
6 & DMAP & 40 & 64 \\
7 & $\mathrm{PPh}_{3}$ & 40 & 59 \\
8 & DBU & 40 & 43 \\
9 & - & 40 & 37 \\
\hline
\end{tabular}

${ }^{a}$ Reactions conditions: aniline $(1 \mathrm{mmol})$, ethyl vinyl ketone $(1.5 \mathrm{mmol})$, catalyst $(30 \mathrm{~mol} \%)$, solvent-free conditions, rt. ${ }^{\mathrm{b}} \mathrm{GC}$ yield. ${ }^{\mathrm{c}}$ Toluene. ${ }^{\mathrm{d}} \mathrm{CH}_{2} \mathrm{Cl}_{2} .{ }^{\mathrm{e}} \mathrm{CH}_{3} \mathrm{OH} .{ }^{\mathrm{f}} \mathrm{CH}_{3} \mathrm{CN}$. ${ }^{\mathrm{g}}$ Reference 6 .

In order to evaluate the generality of the ionic liquid [DBU][Lac] as catalyst for aza-Michael reaction of aromatic amines, 2-cyclohexen-1-one, 2-cyclopenten-1-one and chalcone were tested under the same conditions (Table 2, entries 6-17). To our delight, 2-cyclohexen-1-one and chalcone were effective Michael acceptors to react with various arylamines, achieving good to excellent isolated yields (entries, 6-10, 14-17). However, 2-cyclopenten-1-one showed relatively lower reactivity. Accordingly, higher temperature $\left(60^{\circ} \mathrm{C}\right)$ was required (entries, 11-13). For further comparison with [bmim] $\mathrm{OH}$, [DBU][Lac] was used as promoter for the additions of $p$ methyl aniline to EVK and $p$-methyl aniline to 2-cyclohexen-1-one respectively (entries, 2, 10). With comparable or higher products yields obtained, the two reactions catalyzed by [DBU][Lac] proceeded much faster than those promoted by $[\mathrm{bmim}] \mathrm{OH}$. Moreover, chalcone reacted smoothly with various aromatic amines using [DBU][Lac] as catalyst (entries, 14-17) while no reaction occurred in the presence of the ionic liquid [bmim $] \mathrm{OH} .{ }^{6}$ All cases summarized in Table 2 obviously demonstrate that the novel ionic liquid [DBU][Lac] has the excellent catalytic activity for aza-Michael reaction of aromatic amines and $\alpha, \beta$-unsaturated ketones. 
Table 2. [DBU][Lac] catalyzed aza-Michael reactions of various aromatic amines with $\alpha, \beta$-unsaturated ketones at room temperature ${ }^{\mathrm{a}}$

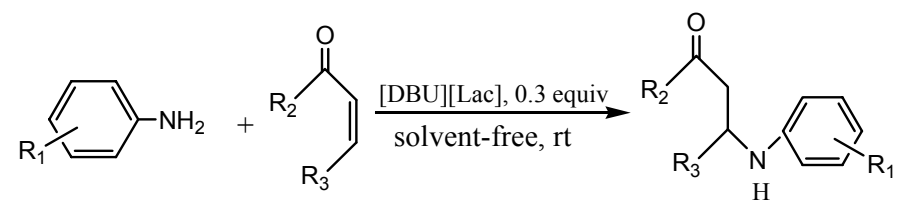

\begin{tabular}{lllllll}
\hline Entry & $\mathrm{R}_{1}$ & $\mathrm{R}_{2}$ & $\mathrm{R}_{3}$ & Time $(\mathrm{h})$ & $\mathrm{GC}$ yield (\%) & Isolated yield (\%) \\
\hline 1 & $4-\mathrm{MeO}$ & $\mathrm{Et}$ & $\mathrm{H}$ & 0.5 & 98 & 95 \\
2 & $4-\mathrm{Me}$ & $\mathrm{Et}$ & $\mathrm{H}$ & $0.1 / 8^{\mathrm{c}}$ & $97 / 98^{\mathrm{c}}$ & 90 \\
3 & $2-\mathrm{Me}$ & $\mathrm{Et}$ & $\mathrm{H}$ & 0.8 & 92 & 85 \\
4 & $4-\mathrm{Cl}$ & $\mathrm{Et}$ & $\mathrm{H}$ & $1 / 8^{\mathrm{c}}$ & $95 / 97^{\mathrm{c}}$ & 90 \\
5 & $2-\mathrm{NO}_{2}$ & $\mathrm{Et}$ & $\mathrm{H}$ & 2 & $\mathrm{NR}$ & $\mathrm{NR}$ \\
6 & $4-\mathrm{H}$ & $\left.-\mathrm{CH}_{2}\right)_{3}$ & & 5 & 89 & 82 \\
7 & $4-\mathrm{Cl}$ & $\left.-\mathrm{CH}_{2}\right)_{3}$ & & 6 & 90 & 79 \\
$8^{\mathrm{b}}$ & $3-\mathrm{Cl}$ & $\left.-\mathrm{CH}_{2}\right)_{3}$ & & 5 & 75 & 68 \\
9 & $2-\mathrm{Me}$ & $\left.-\mathrm{CH}_{2}\right)_{3}$ & & 8 & 85 & 77 \\
10 & $4-\mathrm{Me}$ & $\left.-\mathrm{CH}_{2}\right)_{3}$ & & $5 / 24^{\mathrm{c}}$ & $95 / 71^{\mathrm{c}}$ & 92 \\
$11^{\mathrm{b}}$ & $2-\mathrm{Cl}$ & $\left.-\mathrm{CH}_{2}\right)_{2}$ & & 8 & 70 & 65 \\
$12^{\mathrm{b}}$ & $2-\mathrm{Br}$ & $\left.-\mathrm{CH}_{2}\right)_{2}$ & & 8 & 75 & 69 \\
$13^{\mathrm{b}}$ & $2-\mathrm{Me}$ & $\left.-\mathrm{CH}_{2}\right)_{2}$ & & 8 & 86 & 78 \\
$14^{\mathrm{d}}$ & $4-\mathrm{H}$ & $\mathrm{Ph}$ & $\mathrm{Ph}$ & 5 & 92 & 85 \\
$15^{\mathrm{d}}$ & $4-\mathrm{Cl}$ & $\mathrm{Ph}$ & $\mathrm{Ph}$ & 12 & 75 & 72 \\
$16^{\mathrm{d}}$ & $4-\mathrm{MeO}$ & $\mathrm{Ph}$ & $\mathrm{Ph}$ & 8 & 88 & 83 \\
$17^{\mathrm{d}}$ & $4-\mathrm{Me}$ & $\mathrm{Ph}$ & $\mathrm{Ph}$ & 8 & 91 & 87 \\
\hline
\end{tabular}

${ }^{a}$ Reaction conditions: aromatic amines $(1 \mathrm{mmol}), \alpha, \beta$-unsaturated ketones $(1.5 \mathrm{mmol})$, [DBU][Lac] (30 mol \%), solvent-free conditions, rt. ${ }^{b}$ Reaction conducted at $60{ }^{\circ} \mathrm{C} .{ }^{\mathrm{c}}$ Results obtained from reference 6 , reaction conditions: $[\mathrm{bmim}] \mathrm{OH}$ (0.3 equiv.), aromatic amines (1.0 $\mathrm{mmol})$, acceptors $(1.5 \mathrm{mmol})$, rt, solvent-free. ${ }^{\mathrm{d}} 1.0$ Equiv of [DBU][Lac] required for dissolving chalcone and aromatic amines.

The recyclability of the ionic liquid [DBU][Lac] was then studied using the reaction of aniline and 2-cyclohexen-1-one as a model. The results are shown in Table 3. Upon the completion of the reaction, the reaction solution was extracted with ethyl acetate and purified by flash chromatography. The addition product was identified by ${ }^{1} \mathrm{H}$ NMR, ${ }^{13} \mathrm{C}$ NMR and mass spectra. The residual ionic liquid was washed with ethyl ether, dried under vacuum at $60^{\circ} \mathrm{C}$ for 2 $\mathrm{h}$ and reused for subsequent reactions. As shown in Table 3, the recovered ionic liquid could be used for 8 times without obvious loss of catalytic activity of the ionic liquid. It is worthwhile to 
note that the ionic liquid [DBU][Lac] used for 8 runs remained intact, judging from its ${ }^{1} \mathrm{H}$ NMR spectrum.

Table 3. Recycling and Reusability of the catalyst [DBU][Lac] in the Reaction between aniline and 2-cyclohexen-1-one ${ }^{\mathrm{a}}$

\begin{tabular}{ccc}
\hline Cycle & $\mathrm{t}(\mathrm{h})$ & GC yield $^{\mathrm{b}}(\%)$ \\
\hline 1 & 5 & 89 \\
2 & 5 & 90 \\
3 & 5 & 88 \\
4 & 5 & 89 \\
5 & 5 & 83 \\
6 & 5 & 83 \\
7 & 6 & 90 \\
8 & 6 & 90 \\
\hline
\end{tabular}

${ }^{a}$ Reaction conditions: $1 \mathrm{mmol}$ of aniline, $1.5 \mathrm{mmol}$ of 2-cyclohexen-1-one, $0.3 \mathrm{mmol}$ of [DBU][Lac] without solvents at $\mathrm{rt}^{\mathrm{b}}{ }^{\mathrm{G}} \mathrm{GC}$ yields.

As for the role of DBU-based ionic liquids for the activation in the aza-Michael addition of aromatic amines to $\alpha, \beta$-unsaturated ketones, [DBU][Lac] and [DBU][Ac] were regarded as Brønsted base to promote the addition and [DBU][Tfa] played the role as Brønsted acid for the this type of aza-Michael reaction. The reason for slightly higher catalytic activity of [DBU][Lac] than those of the other two ionic liquids may be the activation of carbonyl group of Michael acceptors by the hydroxyl group at the $\alpha$-position of the carboxyl in lactate anion.

\section{Conclusions}

In conclusion, we have developed three task-specific ionic liquids, [DBU][Lac], [DBU][Ac] and [DBU][Tfa]. The ionic liquids were then used as catalysts for aza-Michael addition of aniline to EVK. Among the three ionic liquids, [DBU][Lac] exhibited the best catalytic activity. Using $[\mathrm{DBU}][\mathrm{Lac}]$ as catalyst, various aromatic amines were subjected to 2-cyclohexen-1-one, 2cyclopenten-1-one and chalcone, and good to excellent yields were obtained. This protocol also has some advantages, such as readily work-up of the reactions and excellent reaction selectivity and good recyclability of the ionic liquid (reused for 8 times without significant loss of activity). 


\section{Experimental Section}

General Procedures. All chemical were purchased from Aldrich or Fluka. ${ }^{1} \mathrm{H}$ and ${ }^{13} \mathrm{C}$ NMR were recorded on a Bruker Avance DPX 400 spectrometer at $400 \mathrm{MHz}$ and $100 \mathrm{MHz}$ in $\mathrm{CDCl}_{3}$ and DMSO- $d_{6}$, respectively. Chemical shifts were reported in parts per million $(\delta)$, relative to the internal standard of tetramethylsilane (TMS). Melting points were determined usingYRT-3 apparatus and were not corrected. Mass spectrometry data were obtained on Brucker Esquire-LC for electro-spay (MS-ES) measurements. Elemental analysis was carried out on a Carlo Erba 1160. All reactions were monitored by thin layer chromatography (TLC). Flash chromatography was performed on silica gel (100-200 mesh). All Michael adducts were purified through column chromatography and were characterised by NMR analysis, melting points and MS.

\section{General procedure for preparation of ionic liquids [DBU][Lac], [DBU][Ac] and [DBU][Tfa].}

DBU $(6 \mathrm{mmol})$ was added to a $50 \mathrm{~mL}$ three -necked flask cooled by ice bath. Carboxylic acid (6 mmol) was then added dropwise at the temperature $\left(\leq 5{ }^{\circ} \mathrm{C}\right)$ with thorough mixing. After dropwise addition, removed the ice bath and the reaction mixture was stirred at room temperature for $24 \mathrm{~h}$. The oil residue was dried in vacuo at $60^{\circ} \mathrm{C}$ for $24 \mathrm{~h}$ to afford desired ionic liquid as light yellow, viscous liquid.

[DBU][Lac]. ${ }^{1} \mathrm{H}$ NMR $\left(400 \mathrm{MHz}, \mathrm{D}_{2} \mathrm{O}\right): \delta=3.93(\mathrm{q}, 1 \mathrm{H}, \mathrm{J}=6.8 \mathrm{~Hz}, \mathrm{CH}), 3.57-3.52(\mathrm{~m}, 2 \mathrm{H}, 9-\mathrm{H})$, 3.47-3.44 (m, 2H, 11-H), 3.28-3..23 (m, 2H, 2-H), 2.73-2.70 (m, 2H, 6-H), 1.93-1.87 (m, 2H, 10$\mathrm{H}), 1.67-1.54(\mathrm{~m}, 6 \mathrm{H}, 3-\mathrm{H}, 4-\mathrm{H}, 5-\mathrm{H}), 1.09\left(\mathrm{~d}, 3 \mathrm{H}, \mathrm{J}=6.8 \mathrm{~Hz}, \mathrm{CH}_{3}\right) ;{ }^{13} \mathrm{C}$ NMR $\left(100 \mathrm{MHz}, \mathrm{D}_{2} \mathrm{O}\right)$ : $\delta=177.8,165.5,67.2,53.4,48.0,36.7,31.5,28.4,26.2,23.7$; Anal. Calcd for [DBU][Lac] C, 59.35; H, 9.21; N, 11.49; O, 19.95. Found C, 59.48; H, 9.15; N, 11.56; O, 19.81.

[DBU][Ac]. ${ }^{1} \mathrm{H}$ NMR $\left(400 \mathrm{MHz}, \mathrm{D}_{2} \mathrm{O}\right): \delta=3.50-3.48(\mathrm{~m}, 2 \mathrm{H}, 9-\mathrm{H}), 3.44-3.41(\mathrm{~m}, 2 \mathrm{H}, 11-\mathrm{H})$, 3.23-3.20 (m, 2H, 2-H), 2.75-2.72 (m, 2H, 6-H), 1.89-1.83 (m, 2H, 10-H), 1.68-1.51 (m, 6H, 3$\mathrm{H}, 4-\mathrm{H}, 5-\mathrm{H}), 1.63$ (s, 3H, $\left.\mathrm{CH}_{3}\right) ;{ }^{13} \mathrm{C}$ NMR $\left(100 \mathrm{MHz}, \mathrm{D}_{2} \mathrm{O}\right): \delta=174.4,165.2,53.1,47.9,37.8$, 31.2, 28.6, 26.5, 25.1, 24.0, 19.5; Anal. Calcd for [DBU][Ac] C, 62.10; H, 9.55; N, 13.11; O, 15.24. Found C, 62.23; H, 9.50; N, 13.20; O, 15.07.

[DBU][Tfa]. ${ }^{1} \mathrm{H}$ NMR (400 MHz, $\left.\mathrm{D}_{2} \mathrm{O}\right): \delta=3.55-3.42(\mathrm{~m}, 8 \mathrm{H}, 9-\mathrm{H}, 11-\mathrm{H}, 2-\mathrm{H}), 2.79-2.77(\mathrm{~m}$, $2 \mathrm{H}, 6-\mathrm{H}), 2.07-2.01(\mathrm{~m}, 2 \mathrm{H}, 10-\mathrm{H}), 1.76-1.70(\mathrm{~m}, 6 \mathrm{H}, 3-\mathrm{H}, 4-\mathrm{H}, 5-\mathrm{H}) ;{ }^{13} \mathrm{C}$ NMR $(100 \mathrm{MHz}$, $\left.\mathrm{D}_{2} \mathrm{O}\right): \delta=166.2,118.4,115.5,54.3,48.5,38.0,32.3,28.9,26.6,23.8,19.3$; Anal. Calcd for [DBU][Tfa] C, 49.54; H, 6.51; N, 10.45; F, 21.37; O, 12.13. Found C, 49.62; H, 6.44; N, 10.52; F, 21.41; O, 12.01.

\section{General procedure for aza-Michael reaction of aliphatic amines with $\alpha, \beta$-unsaturated compounds}

To a mixture of the aromatic amine $(1 \mathrm{mmol})$ and Michael acceptor $(1.5 \mathrm{mmol})$ in $10 \mathrm{~mL}$ flask equipped with a magnetic stirrer was added ionic liquid [DBU][Lac] (0.3 equiv). The reaction mixture was stirred at room temperature for the desired time until the disappearance of starting 
material monitored by TLC. Upon completion of the reaction, the mixture was extracted with ethyl acetate for several times. The combined organic phase was concentrated through vacuum evaporation and the resulting crude product was purified by silica column chromatography to give the desired product. These products are in good agreement with spectra data of literatures. The ionic liquid [DBU][Lac] after extraction was dried in vacuo at $60{ }^{\circ} \mathrm{C}$ for $5 \mathrm{~h}$. The recovered ionic liquid was then reused in subsequent reactions.

1-(Anilinyl)-3-pentanone (Table 1, entry 1). White solid, mp $56-57{ }^{\circ} \mathrm{C}$ (lit., ${ }^{12 \mathrm{a}} 56.2-57.2{ }^{\circ} \mathrm{C}$ ); ${ }^{1} \mathrm{H}$ NMR (400 MHz, $\left.\mathrm{CDCl}_{3}\right): \delta=7.25-7.15(\mathrm{~m}, 2 \mathrm{H}, \mathrm{ArH}), 6.72-6.69(\mathrm{~m}, 1 \mathrm{H}, \mathrm{ArH}), 6.62-6.60(\mathrm{~m}, 2 \mathrm{H}$, ArH), 4.01 (brs, 1H, NH), 3.42 (t, 2H, J=6 Hz, CH ), 2.72 (t, 2H, J=6 Hz, CH $\left.\mathrm{J}=7.2 \mathrm{~Hz}, \mathrm{CH}_{2}\right), 1.05$ (t, 3H, J=7.2 Hz, $\left.\mathrm{CH}_{3}\right) ;{ }^{13} \mathrm{C} \mathrm{NMR}\left(100 \mathrm{MHz}, \mathrm{CDCl}_{3}\right): \delta=210.9,147.6$, 129.2, 117.5, 112.9, 41.1, 38.3, 36.2, 7.6; ESI-MS (m/z): $178(\mathrm{M}+1)$.

1-(4-Methoxylanilinyl)-3-pentanone (Table 2, entry 1). White solid, mp $42-43{ }^{\circ} \mathrm{C}$ (lit., ${ }^{12 b} 41$ $\left.43{ }^{\circ} \mathrm{C}\right) ;{ }^{1} \mathrm{H} \mathrm{NMR}\left(400 \mathrm{MHz}, \mathrm{CDCl}_{3}\right): \delta=6.78(\mathrm{~d}, 2 \mathrm{H}, \mathrm{J}=7.2 \mathrm{~Hz}, \mathrm{ArH}), 6.66(\mathrm{~d}, 2 \mathrm{H}, \mathrm{J}=7.2 \mathrm{~Hz}$, $\mathrm{ArH}), 3.28\left(\mathrm{~s}, 3 \mathrm{H}, \mathrm{OCH}_{3}\right), 3.36\left(\mathrm{t}, 2 \mathrm{H}, \mathrm{J}=6 \mathrm{~Hz}, \mathrm{CH}_{2}\right), 2.71\left(\mathrm{t}, 2 \mathrm{H}, \mathrm{J}=6 \mathrm{~Hz}, \mathrm{CH}_{2}\right), 2.42$ (q, 2H, $\left.\mathrm{J}=7.2 \mathrm{~Hz}, \mathrm{CH}_{2}\right), 1.05\left(\mathrm{t}, 3 \mathrm{H}, \mathrm{J}=7.2 \mathrm{~Hz}, \mathrm{CH}_{3}\right) ;{ }^{13} \mathrm{C} \mathrm{NMR}\left(100 \mathrm{MHz}, \mathrm{CDCl}_{3}\right): \delta=211.1,152.1$, 141.8, 114.7, 114.5, 55.6, 41.2, 39.5, 36.2, 7.6; ESI-MS (m/z): $208(\mathrm{M}+1)$.

1-(p-Toluidinyl)-3-pentanone (Table 2, entry 2). White solid, mp 64-65 ${ }^{\circ} \mathrm{C} ;{ }^{1} \mathrm{H}$ NMR (400 $\left.\mathrm{MHz}_{2} \mathrm{CDCl}_{3}\right): \delta=6.98(\mathrm{~d}, 2 \mathrm{H}, \mathrm{J}=8.0 \mathrm{~Hz}, \mathrm{ArH}), 6.53$ (d, 2H, J=8.0 Hz, ArH), 3.88 (brs, 1H, NH), 3.39 (t, 2H, J=6 Hz, CH 2 ), 2.70 (t, 2H, J=6 Hz, CH ), 2.43 (q, 2H, J=7.2 Hz, CH $\left.\mathrm{CH}_{3}\right), 1.04\left(\mathrm{t}, 3 \mathrm{H}, \mathrm{J}=7.2 \mathrm{~Hz}, \mathrm{CH}_{3}\right) ;{ }^{13} \mathrm{C} \mathrm{NMR}\left(100 \mathrm{MHz}, \mathrm{CDCl}_{3}\right): \delta=211.0,145.3,129.7,126.8$, 113.2, 41.2, 38.8, 36.2, 20.3, 7.6; ESI-MS (m/z): $192(\mathrm{M}+1)$.

1-(o-Toluidinyl)-3-pentanone (Table 2, entry 3). Pale yellow oil; ${ }^{1} \mathrm{H}$ NMR (400 $\left.\mathrm{MHz}, \mathrm{CDCl}_{3}\right)$ : $\delta=7.13(\mathrm{t}, 2 \mathrm{H}, \mathrm{J}=8.0 \mathrm{~Hz}, \operatorname{ArH}), 7.05$ (d, 2H, J=7.2 Hz, ArH), 6.68-6.61 (m, 2H, ArH), 3.94 (brs, 1H, NH), 3.47 (t, 2H, J=6 Hz, CH 2$), 2.75$ (t, 2H, J=6 Hz, $\left.\mathrm{CH}_{2}\right), 2.43$ (q, 2H, J=7.2 Hz, $\mathrm{CH}_{2}$ ), 2.11 (s, 3H, $\left.\mathrm{CH}_{3}\right), 1.03$ (t, 3H, J=7.2 Hz, $\left.\mathrm{CH}_{3}\right) ;{ }^{13} \mathrm{C} \mathrm{NMR}\left(100 \mathrm{MHz}, \mathrm{CDCl}_{3}\right): \delta=211.0,145.6,130.2$, 127.0, 122.5, 117.0, 109.5, 41.1, 38.3, 36.3, 17.4, 7.6; ESI-MS (m/z): $192(\mathrm{M}+1)$.

1-(4- Chloroanilinyl)-3-pentanone (Table 2, entry 4). White solid, mp 74-76 ${ }^{\circ} \mathrm{C} ;{ }^{1} \mathrm{H}$ NMR (400 $\mathrm{MHz}_{\mathrm{CDCl}}$ ): $\delta=7.12$ (d, 2H, J=8.8 Hz, ArH), 6.53 (d, 2H, J=8.8 Hz, ArH), 4.07 (brs, 1H, NH), 3.39 (t, 2H, J=6 Hz, CH ), 2.72 (t, 2H, J=6 Hz, CH ), 2.45 (q, 2H, J=7.2 Hz, CH $\left.\mathrm{J}=7.2 \mathrm{~Hz}, \mathrm{CH}_{3}\right) ;{ }^{13} \mathrm{C} \mathrm{NMR}\left(100 \mathrm{MHz}, \mathrm{CDCl}_{3}\right): \delta=210.8,146.2,129.0,121.9,114.0,40.9,38.4$, 36.37.6; ESI-MS (m/z): $212(\mathrm{M}+1)$.

3-(Anilinyl)-cyclohexan-1-one (Table 2, entry 6). Pale yellow oil; ${ }^{1} \mathrm{H}$ NMR (400 MHz, $\mathrm{CDCl}_{3}$ ): $\delta=7.19-7.13$ (m, 3H, ArH), 6.77-6.69 (m, 2H, ArH), 3.78 (brs, 1H, NH), 2.83 (m, 1H, $\mathrm{CH}), 2.43-2.15\left(\mathrm{~m}, 4 \mathrm{H}, 2 \times \mathrm{CH}_{2}\right), 2.09-1.99\left(\mathrm{~m}, 2 \mathrm{H}, \mathrm{CH}_{2}\right), 1.79-1.64\left(\mathrm{~m}, 2 \mathrm{H}, \mathrm{CH}_{2}\right) ;{ }^{13} \mathrm{C} \mathrm{NMR}$ $\left(100 \mathrm{MHz} \mathrm{CDCl}_{3}\right): \delta=209.4,146.0,129.1,117.6,113.0,52.0,48.2$, 40.8, 30.7, 21.8; ESI-MS $(\mathrm{m} / \mathrm{z}): 190(\mathrm{M}+1)$.

3-(4-Chloroanilinyl)-cyclohexan-1-one (Table 2, entry 7). Pale yellow oil; ${ }^{1} \mathrm{H}$ NMR (400 $\left.\mathrm{MHz}, \mathrm{CDCl}_{3}\right): \delta=7.11(\mathrm{~d}, 2 \mathrm{H}, \mathrm{J}=8 \mathrm{~Hz}, \mathrm{ArH}), 6.50$ (d, 2H, J=8 Hz, ArH), 3.74 (brs, 1H, NH), 2.83-2.81 (m, 1H, CH), 2.43-2.23 (m, 4H, 2× $\left.\mathrm{CH}_{2}\right), 2.20-2.00\left(\mathrm{~m}, 2 \mathrm{H}, \mathrm{CH}_{2}\right), 1.77-1.62(\mathrm{~m}, 2 \mathrm{H}$, 
$\left.\mathrm{CH}_{2}\right) ;{ }^{13} \mathrm{C} \mathrm{NMR}\left(100 \mathrm{MHz}, \mathrm{CDCl}_{3}\right): \delta=209.6,145.1,129.5,122.6,114.6,52.6,48.5,41.3,31.1$, 22.3; ESI-MS (m/z): $224(\mathrm{M}+1)$.

3-(3-Chloroanilinyl)-cyclohexan-1-one (Table 2, entry 8). Pale yellow oil; ${ }^{1} \mathrm{H}$ NMR (400 $\left.\mathrm{MHz}, \mathrm{CDCl}_{3}\right): \delta=7.10-7.03(\mathrm{~m}, 1 \mathrm{H}, \mathrm{ArH}), 6.71-6.66(\mathrm{~m}, 1 \mathrm{H}, \mathrm{ArH}), 6.55-6.49(\mathrm{~m}, 1 \mathrm{H}, \mathrm{ArH})$, 6.46-6.43 (m, 1H, ArH), 3.64 (brs, $1 \mathrm{H}, \mathrm{NH}), 2.83-2.80(\mathrm{~m}, 1 \mathrm{H}, \mathrm{CH}), 2.44-2.25\left(\mathrm{~m}, 4 \mathrm{H}, 2 \times \mathrm{CH}_{2}\right.$ ), 2.17-2.01 (m, 2H, $\left.\mathrm{CH}_{2}\right), 1.81-1.71\left(\mathrm{~m}, 2 \mathrm{H}, \mathrm{CH}_{2}\right) ;{ }^{13} \mathrm{C} \mathrm{NMR}\left(100 \mathrm{MHz}, \mathrm{CDCl}_{3}\right): \delta=209.3,147.3$, 135.1, 130.3, 117.6, 112.7, 111.5, 52.0, 48.2, 41.0, 30.8, 22.0; ESI-MS (m/z): $224(\mathrm{M}+1)$.

3-(o-Toluidinyl)-cyclohexan-1-one (Table 2, entry 9). Pale yellow oil; ${ }^{1} \mathrm{H}$ NMR (400 MHz, $\left.\mathrm{CDCl}_{3}\right): \delta=7.14-7.06(\mathrm{~m}, 2 \mathrm{H}, \mathrm{ArH}), 6.70-6.66(\mathrm{~m}, 2 \mathrm{H}, \mathrm{ArH}), 6.62-6.60(\mathrm{~m}, 1 \mathrm{H}, \mathrm{ArH}), 3.44$ (brs, $1 \mathrm{H}, \mathrm{NH}), 2.89-2.85(\mathrm{~m}, 1 \mathrm{H}, \mathrm{CH}), 2.42-2.28\left(\mathrm{~m}, 4 \mathrm{H}, 2 \times \mathrm{CH}_{2}\right), 2.12\left(\mathrm{~s}, 3 \mathrm{H}, \mathrm{CH}_{3}\right), 2.08-2.01(\mathrm{~m}$, $\left.2 \mathrm{H}, \mathrm{CH}_{2}\right), 1.82-1.70\left(\mathrm{~m}, 2 \mathrm{H}, \mathrm{CH}_{2}\right) ;{ }^{13} \mathrm{C} \mathrm{NMR}\left(100 \mathrm{MHz}, \mathrm{CDCl}_{3}\right): \delta=209.7,144.1,130.4,127.1$, 122.0, 117.3, 110.2, 52.0, 48.6, 41.1, 38.0, 31.0, 22.1, 17.5; ESI-MS (m/z): $204(\mathrm{M}+1)$.

3-(p-Toluidinyl)-cyclohexan-1-one (Table 2, entry 10). Pale yellow oil; ${ }^{1} \mathrm{H}$ NMR (400 MHz, $\left.\mathrm{CDCl}_{3}\right): \delta=6.98(\mathrm{~d}, 2 \mathrm{H}, \mathrm{J}=8 \mathrm{~Hz}, \mathrm{ArH}), 6.51(\mathrm{~d}, 2 \mathrm{H}, \mathrm{J}=8 \mathrm{~Hz}, \mathrm{ArH}), 3.73$ (brs, $\left.1 \mathrm{H}, \mathrm{NH}\right), 2.82-2.78$ (m, $1 \mathrm{H}, \mathrm{CH}), 2.43-2.24\left(\mathrm{~m}, 4 \mathrm{H}, 2 \times \mathrm{CH}_{2}\right), 2.16-2.03\left(\mathrm{~m}, 2 \mathrm{H}, \mathrm{CH}_{2}\right), 1.72-1.67\left(\mathrm{~m}, 2 \mathrm{H}, \mathrm{CH}_{2}\right) ;{ }^{13} \mathrm{C}$ NMR (100 MHz, $\left.\mathrm{CDCl}_{3}\right): \delta=210.1,144.2,130.1,127.4,113.8,52.9,48.9,41.4,31.3,22.4,20.6$; ESI-MS (m/z): $204(\mathrm{M}+1)$.

3-(2-Chloroanilinyl)-cyclopentan-1-one (Table 2, entry 11). Pale yellow oil; ${ }^{1} \mathrm{H}$ NMR (400 $\left.\mathrm{MHz}, \mathrm{CDCl}_{3}\right): \delta=7.28(\mathrm{~m}, 1 \mathrm{H}, \mathrm{ArH}), 7.16(\mathrm{~m}, 1 \mathrm{H}, \mathrm{ArH}), 6.68(\mathrm{~m}, 2 \mathrm{H}, \mathrm{ArH}), 4.36$ (brs, $\left.1 \mathrm{H}, \mathrm{NH}\right)$, $4.15(\mathrm{~m}, 1 \mathrm{H}, \mathrm{CH}), 2.77-2.70\left(\mathrm{~m}, 2 \mathrm{H}, \mathrm{CH}_{2}\right), 2.53-2.46\left(\mathrm{~m}, 2 \mathrm{H}, \mathrm{CH}_{2}\right), 2.45-2.19\left(\mathrm{~m}, 2 \mathrm{H}, \mathrm{CH}_{2}\right) ;{ }^{13} \mathrm{C}$ NMR (100 MHz, $\left.\mathrm{CDCl}_{3}\right): \delta=218.4,142.7,129.3,127.8,119.4,117.9,111.7,50.9,45.8,36.6$, 29.7; ESI-MS (m/z): $210(\mathrm{M}+1)$.

3-(2-Bromoanilinyl)-cyclopentan-1-one (Table 2, entry 12). Pale yellow oil; ${ }^{1} \mathrm{H}$ NMR (400 $\left.\mathrm{MHz}, \mathrm{CDCl}_{3}\right): \delta=7.45-7.39(\mathrm{~m}, 1 \mathrm{H}, \mathrm{ArH}), 7.22-7.17(\mathrm{~m}, 1 \mathrm{H}, \mathrm{ArH}), 6.67-6.60(\mathrm{~m}, 2 \mathrm{H}, \mathrm{ArH}), 4.37$ (brs, $1 \mathrm{H}, \mathrm{NH}), 4.14(\mathrm{~m}, 1 \mathrm{H}, \mathrm{CH}), 2.77-2.69\left(\mathrm{~m}, 2 \mathrm{H}, \mathrm{CH}_{2}\right), 2.53-2.45\left(\mathrm{~m}, 2 \mathrm{H}, \mathrm{CH}_{2}\right), 2.44-2.19(\mathrm{~m}$, $\left.2 \mathrm{H}, \mathrm{CH}_{2}\right) ;{ }^{13} \mathrm{C} \mathrm{NMR}\left(100 \mathrm{MHz}, \mathrm{CDCl}_{3}\right): \delta=216.4,143.7,132.6,128.5,118.5,111.8,110.1,51.1$, 45.8, 36.6, 29.7; ESI-MS (m/z): $255(\mathrm{M}+1)$.

3-( o-Toluidinyl)-cyclopentan-1-one (Table 2, entry 13). Pale yellow oil; ${ }^{1} \mathrm{H}$ NMR (400 MHz, $\left.\mathrm{CDCl}_{3}\right): \delta=7.16-7.02(\mathrm{~m}, 2 \mathrm{H}, \mathrm{ArH}), 6.73-6.65(\mathrm{~m}, 2 \mathrm{H}, \mathrm{ArH}), 4.19-4.10(\mathrm{~m}, 1 \mathrm{H}, \mathrm{CH}), 3.57$ (brs, $1 \mathrm{H}, \mathrm{NH}), 2.77-2.68\left(\mathrm{~m}, 2 \mathrm{H}, \mathrm{CH}_{2}\right), 2.51-1.97\left(\mathrm{~m}, 4 \mathrm{H}, 2 \times \mathrm{CH}_{2}\right), 2.44-2.19\left(\mathrm{~m}, 2 \mathrm{H}, \mathrm{CH}_{2}\right) ;{ }^{13} \mathrm{C} \mathrm{NMR}$ $\left(100 \mathrm{MHz}, \mathrm{CDCl}_{3}\right): \delta=217.1,144.7,134.5,130.3,127.0,117.6,110.4 ;$ ESI-MS $(\mathrm{m} / \mathrm{z}): 190$ $(\mathrm{M}+1)$.

$\mathrm{N}$-(3-Oxo-1,3-diphenylpropyl)aniline (Table 2, entry 14). White solid, mp $173-175{ }^{\circ} \mathrm{C}$ (lit., ${ }^{12 \mathrm{c}}$ 172-174 $\left.{ }^{\circ} \mathrm{C}\right) ;{ }^{1} \mathrm{H}$ NMR $\left(400 \mathrm{MHz}, \mathrm{CDCl}_{3}\right): \delta=7.92(\mathrm{~d}, 2 \mathrm{H}, \mathrm{J}=8.0 \mathrm{~Hz}, \mathrm{ArH}), 7.56(\mathrm{t}, 1 \mathrm{H}, \mathrm{J}=7.2 \mathrm{~Hz}$, ArH), 7.46-7.43 (m, 4H, ArH), 7.33 (t, 2H, J=7.6 Hz, ArH), 7.24 (t, 1H, J=7.6 Hz, ArH), 7.10 (t, $2 \mathrm{H}, \mathrm{J}=8.0 \mathrm{~Hz}, \mathrm{ArH}), 6.67$ (t, 1H, J=7.2 Hz, ArH), 6.57 (d, 2H, J=8.0 Hz, ArH), 5.02 (brs, 1H, $\mathrm{NH}), 4.58-4.57(\mathrm{~m}, 1 \mathrm{H}, \mathrm{CH}), 3.55-3.40\left(\mathrm{~m}, 2 \mathrm{H}, \mathrm{CH}_{2}\right) ;{ }^{13} \mathrm{C} \mathrm{NMR}\left(100 \mathrm{MHz}, \mathrm{CDCl}_{3}\right): \delta=198.2$, 146.9, 142.9, 136.5, 133.4, 129.0, 128.8, 128.7, 128.2, 127.3, 126.3, 117.7, 113.7, 54.6, 46.3; ESI-MS (m/z): $302(\mathrm{M}+1)$. 
$\boldsymbol{N}$-(3-Oxo-1,3-diphenylpropyl)-4-chloroaniline (Table 2, entry 15). White solid, mp 168-170 ${ }^{\circ} \mathrm{C}$ (lit., ${ }^{12 \mathrm{~d}} 169-170{ }^{\circ} \mathrm{C}$ ); ${ }^{1} \mathrm{H}$ NMR (400 MHz, $\mathrm{CDCl}_{3}$ ): $\delta=7.90$ (d, 2H, J=7.2 Hz, ArH), $7.56(\mathrm{t}, 1 \mathrm{H}, \mathrm{J}=7.6 \mathrm{~Hz}, \operatorname{ArH}), 7.46-7.40(\mathrm{~m}, 4 \mathrm{H}, \mathrm{ArH}), 7.32(\mathrm{t}, 2 \mathrm{H}, \mathrm{J}=7.6 \mathrm{~Hz}, \mathrm{ArH}), 7.24(\mathrm{t}, 1 \mathrm{H}$, $\mathrm{J}=7.6 \mathrm{~Hz}, \operatorname{ArH}), 7.01$ (d, 2H, J=8.8 Hz, ArH), 6.47 (d, 2H, J=8.8 Hz, ArH), 4.94 (brs, 1H, NH), 4.54-4.52 (m, 1H, CH), 3.52-3.38 (m, $\left.2 \mathrm{H}, \mathrm{CH}_{2}\right) ;{ }^{13} \mathrm{C}$ NMR (100 MHz, $\left.\mathrm{CDCl}_{3}\right): \delta=198.5,145.8$, $142.8,136.8,133.8,129.2,129.0,128.5,127.8,126.6,122.7,115.2,55.1,46.5 ;$ ESI-MS (m/z): $336(\mathrm{M}+1)$.

N-(3-Oxo-1,3-diphenylpropyl)-4-methoxyaniline (Table 2, entry 16). White solid, mp 142$143{ }^{\circ} \mathrm{C}$ (lit., $\left.{ }^{12 \mathrm{e}} 140-144{ }^{\circ} \mathrm{C}\right) ;{ }^{1} \mathrm{H}$ NMR $\left(400 \mathrm{MHz}, \mathrm{CDCl}_{3}\right): \delta=7.90(\mathrm{~d}, 2 \mathrm{H}, \mathrm{J}=7.6 \mathrm{~Hz}, \mathrm{ArH}), 7.55(\mathrm{t}$, 1H, J=7.2 Hz, ArH), 7.43-7.42 (m, 4H, ArH), 7.31 (t, 2H, J=7.2 Hz, ArH), $7.22(\mathrm{t}, 1 \mathrm{H}, \mathrm{J}=7.2 \mathrm{~Hz}$, $\operatorname{ArH}), 6.67$ (d, 2H, J=8.4 Hz, ArH), 6.52 (d, 2H, J=8.4 Hz, ArH), 4.92 (brs, 1H, NH), 4.23 (m, $1 \mathrm{H}, \mathrm{CH}), 3.68\left(\mathrm{~s}, 3 \mathrm{H}, \mathrm{CH}_{3}\right), 3.50-3.36\left(\mathrm{~m}, 2 \mathrm{H}, \mathrm{CH}_{2}\right) ;{ }^{13} \mathrm{C} \mathrm{NMR}\left(100 \mathrm{MHz}, \mathrm{CDCl}_{3}\right): \delta=198.3$, $152.3,143.2,141.2,136.7,133.4,128.8,128.7,128.2,127.3,126.4,115.3,114.7,55.6,46.4$, 39.9; ESI-MS (m/z): $332(\mathrm{M}+1)$.

N-(3-Oxo-1,3-diphenylpropyl)-4-methylaniline (Table 2, entry 17) White solid, mp 170-172 ${ }^{\circ} \mathrm{C}$ (lit., $\left.{ }^{12 \mathrm{c}} 170-171{ }^{\circ} \mathrm{C}\right)$; ${ }^{1} \mathrm{H}$ NMR $\left(400 \mathrm{MHz}, \mathrm{CDCl}_{3}\right): \delta=7.94-7.89$ (m, 2H, ArH), 7.55 (t, 1H, $\mathrm{J}=7.2 \mathrm{~Hz}, \operatorname{ArH}), 7.43-7.42(\mathrm{~m}, 4 \mathrm{H}, \operatorname{ArH}), 7.32-7.22(\mathrm{~m}, 4 \mathrm{H}, \operatorname{ArH}), 6.89$ (d, 2H, J=7.2 Hz, ArH), 6.47 (d, 2H, J=7.2 Hz, ArH), 4.96 (brs, 1H, NH), 4.43-4.41 (m, 1H, CH), 3.52-3.37 (m, 2H, $\left.\mathrm{CH}_{2}\right), 2.17\left(\mathrm{~s}, 3 \mathrm{H}, \mathrm{CH}_{3}\right) ;{ }^{13} \mathrm{C} \mathrm{NMR}\left(100 \mathrm{MHz}, \mathrm{CDCl}_{3}\right): \delta=198.3,144.7,143.1,136.7,133.4$, $129.6,129.0,128.8,128.7,128.2,127.3,126.4,114.0,55.0,46.3,20.3 ;$ ESI-MS (m/z): 316 $(\mathrm{M}+1)$.

\section{Acknowledgements}

This work was supported by the National Key Technology R\&D Program (No.2007BAI34B07) and the Natural Science Foundation of China (NO. 20776127).

\section{References}

1. (a) Wang, Y.-F.; Izawa, T.; Kobayashi, S.; Ohno, M. J. Am. Chem. Soc. 1982, 104, 6465. (b) Eliel, E. L.; He, X. C. J. Org. Chem. 1990, 55, 2114. (c) Hayashi, Y.; Rode, J. J.; Corey, E. J. J. Am. Chem. Soc. 1996, 118, 5502. (d) Cardillo, G.; Tomasini, C. Chem. Soc. Rev. 1996, 117. (e) Fustero, S.; Pina, B.; Salavert, E.; Navarro, A.; Ramirez de Arellano, M. C.; Fuentes, A. S. J. Org. Chem. 2002, 67, 4667.

2. (a) Kobayashi, S.; Kakumoto, K.; Sugiura, M. Org. Lett. 2002, 4, 1319. (b) Xu, L.-W.; Li, J.W.; Xia, C.-G., Zhou, S.-L.; Hu, X.-X. Synlett 2003, 2425. (c) Loh, T.P.; Wei, L.-L. Synlett 1998, 975. (d) Matsubara, S.; Yoshiyoka, M.; Utimoto, K. Chem. Lett. 1994, 827. (e) Jenner, G. Tetrahedron Lett. 1995, 36, 233. (f) Azizi, N.; Said, M. R. Tetrahedron 2004, 60, 383. (g) 
Srivastava, N.; Banik, B. K. J. Org. Chem. 2003, 68, 2109. (h) Xu, L.-W.; Li, L.; Xia, C.-G. Helv. Chim. Acta. 2004, 87, 1522. (i) Bartoli, G; Bosco, M.; Marcantoni, E.; Petrini, M.; Sanbri, L.; Torregiani, E. J. Org. Chem. 2001, 66, 9052. (j) Zare, A.; Hasaninejad, A.; Khalafi-Nezhad, A.; Zare, A. R. M.; Parhami, A.; Nejabat, G. R. Arkivoc 2007, (i), 58. (k) Zare, A.; Hasaninejad, A.; Khalafi-Nezhad, A.; Zare, A. R. M.; Parhami, A. Arkivoc 2007, (xiii), 105. (1) Mukherjee, C.; Misra, A. K. Lett. Org. Chem. 2007, 4, 54. (m) Reddy, B. M.; Patil, M. K.; Reddy, B. T. Catal. Lett. 2008, 126, 413.

3. (a) You, L.-S.; Feng, S.; An, R.; Wang, X.-H.; Bai, D.-L. Tetrahedron Lett. 2008, 49, 5147.

(b) Surendra, K.; Krishnaveni, N. S.; Sridhar, R.; Rao, K. R. Tetrahedron Lett. 2006, 47, 2125. (c) Kumar, R.; Chaudhary, P.; Nimesh, S.; Chandra, R. Green Chem. 2006, 8, 356. (d) Sharma, Y. O.; Degani, M. S. J. Mol. Catal. A: Chem. 2007, 277, 215. (e) Chaudhuri, M. K.; Hussain, S.; Kantam, M. L.; Neelima, B. Tetrahedron Lett. 2005, 46, 8329. (f) Ranu, B. C.; Banerjee, S. Tetrahedron Lett. 2007, 48, 141.

4. Duan, Z.; Xuan, X.-J.; Li, T.; Yang, C.-F.; Wu, Y.-J. Tetrahedron Lett. 2006, 47, 5433.

5. Bhanushali, M. J.; Nandurkar, N. S.; Jagtap, S. R.; Bhanage, B. M. Catal. Commun. 2008, 9, 1189.

6. Yang, L.; Xu, L.-W.; Zhou, W.; Li, L.; Xia, C.-G. Tetrahedron Lett. 2006, 47, 7723.

7. (a) Reed, R.; Réau, R.; Dahan, F.; Bertrand, G. Angew. Chem. Int. Ed. 1993, 32, 399. (b) Baidya, M.; Mayr, H. Chem. Commun. 2008, 1792. (c) Ghosh, N. Synlett 2004, 574 and references there cited in.

8. (a) Yeom, C.-E.; Kim, M.-J.; Kim, B.-M. Tetrahedron 2007, 63, 904. (b) Han, X.-J. Tetrahedron Lett. 2007, 48, 2845.

9. (a) Welton, T. Chem Rev. 1999, 99, 2071. (b) Miao, W.-W.; Chan, T.H. Acc. Chem. Res. 2006, 39, 897. (c) Ying, A.-G.; Ye, W.-D.; Liu, L.; Wu, G.-F.; Chen, X.-Z.; Qian, S.; Zhang, Q.-P. Chinese J. Org. Chem. 2008, 28, 2081 and references there cited in.

10. Tolstikova, L. L.; Shainyan, B. A. Russian J. Org. Chem. 2006, 42, 1068.

11. (a) Ranu, B. C.; Banerjee, S. Org. Lett. 2005, 7, 3049. (b) Xu, J.-M.; Wu, Q.; Zhang, Q.-Y.; Zhang, F.; Lin, X.-F. Eur. J. Org. Chem. 2007, 1798

12. (a) Archer, S.; Dickinson, W.; Unser, M. J. Org. Chem. 1957, 22, 92. (b) Hughes, G.; Kimura, M.; Buchwald, S. L. J. Am. Chem. Soc. 2003, 125, 11253. (c) José, B.; Humildad, C.; Bernardo, O.; Santos, F.; Vicente, G. Synthesis 1986, 469. (d) Wang, R.; Li, B. G.; Huang, T. K.; Shi, L.; Lu, X. X. Tetrahedron Lett. 2007, 48, 2071. (e) Zahouily, M.; Bahlaouan, W.; Bahlaouan, B.; Rayadh, A.; Sebti, S. Arkivoc 2005, (xiii), 150. 